\title{
Association of surgical interval and survival among hospital and non-hospital based patients with melanoma in North Carolina
}

\author{
Adewole S. Adamson ${ }^{1,2,3}$ - Bradford E. Jackson ${ }^{4}$. Christopher D. Baggett ${ }^{4,5} \cdot$ Nancy E. Thomas $^{3,4}$. \\ Michael P. Pignone ${ }^{1,2}$
}

Received: 1 July 2020 / Revised: 30 August 2020 / Accepted: 9 October 2020 / Published online: 23 October 2020

(c) Springer-Verlag GmbH Germany, part of Springer Nature 2020

\begin{abstract}
Surgical excision is important for melanoma treatment. Delays in surgical excision after diagnosis of melanoma have been linked to decreased survival in hospital-based cohorts. This study was aimed at quantifying the association between the timeliness of surgical excision and overall survival in patients diagnosed with melanoma in hospital- and non-hospitalbased settings, using a retrospective cohort study of patients with stage 0-III melanoma and using data linked between the North Carolina Central Cancer Registry to Medicare, Medicaid, and private health insurance plan claims across the state. We identified 6,496 patients diagnosed between 2004 and 2012 with follow-up through 2017. We categorized the time from diagnostic biopsy to surgical excision as $<6$ weeks after diagnosis, 6 weeks to 90 days after diagnosis, and $>90$ days after melanoma diagnosis. Multivariable Cox regression was used to estimate differences in survival probabilities. Five-year overall survival was lower for those with time to surgery over 90 days (78.6\%) compared with those with less than 6 weeks $(86 \%)$. This difference appeared greater for patients with Stage 1 melanoma. This study was retrospective, included one state, and could not assess melanoma specific mortality. Surgical timeliness may have an effect on overall survival in patients with melanoma. Timely surgery should be encouraged.
\end{abstract}

Keywords Melanoma $\cdot$ Surgery $\cdot$ Delays $\cdot$ Quality of care $\cdot$ Insurance $\cdot$ Medicare $\cdot$ Medicaid $\cdot$ Private insurance $\cdot$ Disparities

\begin{abstract}
Abbreviations
CIPHR Cancer information and population health resource

NCCCR North Carolina Central Cancer Registry
\end{abstract}

Electronic supplementary material The online version of this article (https://doi.org/10.1007/s00403-020-02146-2) contains supplementary material, which is available to authorized users.

Adewole S. Adamson

adewole.adamson@austin.utexas.edu

1 Department of Internal Medicine (Division of Dermatology), Dell Medical School at The University of Texas At Austin, 1601 Trinity St., Stop Z0900, Austin, TX 78712, USA

2 LIVESTRONG Cancer Institutes, The University of Texas at Austin, Austin, TX, USA

3 Department of Dermatology, University of North Carolina at Chapel Hill, Chapel Hill, NC, USA

4 Lineberger Comprehensive Cancer Center, University of North Carolina at Chapel Hill, Chapel Hill, NC, USA

5 Department of Epidemiology, University of North Carolina at Chapel Hill, Chapel Hill, NC, USA
AJCC American joint committee on cancer

NCI National cancer institute

\section{Introduction}

Nearly 100,000 people were diagnosed with melanoma in 2019. Based on Surveillance, Epidemiology, and End Results (SEER) data, the incidence of invasive melanoma increased more than threefold over the past 40 year [1]. Melanoma is responsible for over 7,000 deaths annually, and it is estimated that patients with melanoma potentially lose an average of over 20 years of life as a result of melanoma mortality [2]. Timely surgical excision is an important part of melanoma treatment and, depending on stage of disease, is often the definitive intervention. Recently, the COVID19 pandemic has resulted in delays in cancer surgery, particularly for early stage cancers, which may have important implications for quality and equity of care.

In cancer, delays in treatment can result in increased morbidity and mortality. For example, in breast cancer, shortening of delays to surgery has been associated with benefits 
comparable to some standard treatments [3]. Previous studies in melanoma have revealed that surgical delays greater than 6 weeks, the suggested upper limit of standard of care, between diagnosis and surgery are common and disproportionately affect non-white and low-income patients, who are also at increased risk of poor clinical outcomes [4-7]. These potentially preventable delays longer than 6 weeks without definitive treatment could result in tumor progression and worse survival outcomes [8]. While delays are a metric used to assess quality of care received, less is known about how these delays affect survival. Small studies assessing the association between timeliness of surgery and survival found no effect on survival $[9,10]$. In contrast, a recent U.S. national study of patients with melanoma showed that a delay in surgery of 90 days or more had a negative impact on overall survival; however, this study included only patients with melanomas diagnosed in hospitals, whereas most melanomas are diagnosed in the outpatient setting [11]. Our study sought to address this gap in knowledge.

Using a unique data set linking data from a state-wide cancer registry with insurance claims, we sought to quantify the association between surgical delay and overall survival among a diverse, insured population with widely varying access to care. In addition, we examined whether the relationship between delays in care and survival differs by initial stage at diagnosis.

\section{Methods}

\section{Data source and study population}

This was a retrospective cohort study of patients with incident stage 0-III melanoma. The data were obtained from the University of North Carolina Cancer Information Population Health Resource (CIPHR), a data resource that links cancer data from the North Carolina Central Cancer Registry to administrative and claims data from Medicare, Medicaid, and private health insurance plans across the state [12]. These data cover $70 \%$ of the cancer patients in North Carolina. We included patients aged 20-100 years whose only cancer diagnosis was melanoma. Patients were diagnosed between 2004 and 2012 and were followed for up to 5 years after diagnosis through 2017. Eligible patients were included if they had continuous enrollment in their insurance plan from 6 months prior through 12 months after diagnosis. Patients were included if they had a skin biopsy, defined as either an excision, skin biopsy, or shave removal procedure code, occurring within 30 days before or 7 days after melanoma diagnosis. Patients were excluded if their diagnosis was only identified from death certificate or autopsy. In addition, patients were excluded if they did not undergo an excision within the first year after being diagnosed with melanoma. Moreover, patients with Stage IV tumors were excluded from this analysis because delays could have been due to inoperability. Observations with missing or incomplete data for biopsy, excision, or stage at diagnoses were excluded from the analytic sample. The University of North Carolina Institutional Review Board approved the study. Informed consent was waived due to the retrospective nature of the study.

\section{Variables}

The primary outcome was overall survival, defined as the time from surgical excision to death from any cause. Survival data were obtained from data in the North Carolina Central Cancer Registry through 2017, which allowed assessment of survival at 1,3 , and 5 years after diagnosis; patients were censored if no event was observed within 5 years of follow-up. Our primary explanatory variable was surgical delay, defined as the time between biopsy and definitive surgical excision and categorized into the following categories: $<6$ weeks after diagnosis, 6 weeks to 90 days after diagnosis, and $>90$ days after melanoma diagnosis. We excluded surgical delays beyond 365 days due to small number of cases. Potential confounders of the treatment-outcome relation included the following: Age at diagnosis, sex, race/ethnicity, American Joint Committee on Cancer (AJCC) 6th edition clinical stage of disease, tumor site, insurance coverage at diagnosis, urban vs. rural residence, year of diagnosis, specialty of the physician making the diagnosis (dermatologist vs. other), excision facility (North Carolina National Cancer Institute center vs. other), and comorbid conditions. Stage of disease was categorized as stages 0 , IA, IB, II, and III; tumor site as head/neck, upper extremities, lower extremities, trunk, and other skin not otherwise specified; and insurance coverage at diagnosis as having any private, Medicare only, and any Medicaid. Residence was dichotomized as rural and urban based on the Rural Urban Commuting Area codes [13]. Comorbidities were assessed using the Charlson Comorbidity Index and were categorized as 0 vs. 1 or more comorbid condition present during the 6 months prior to diagnosis.

\section{Data analysis}

We estimated frequencies and proportions for categorical variables, and medians and interquartile ranges for continuous variables. Between-group differences in proportions and medians were assessed with chi-square tests and Kruskal-Wallis one-way analysis of variance, respectively. To estimate the effect of surgical delay on overall survival, we estimated adjusted survival probabilities from multivariable Cox regression models. We calculated survival differences and corresponding $95 \%$ confidence limits as 
the difference between surgical delay grouping's survival probabilities at 1,3 , and 5 years of follow-up. The reference group comprised patients who received surgical excision $<6$ weeks after diagnosis. The proportional hazards assumption was violated; consequently, we were not able to present estimates of the hazard ratio. Age at diagnosis was treated as a restricted quadratic spline with knots at the 5th, 35th, 65th, and 95th percentiles in our statistical model [14]. Moreover, to allow for flexible modeling, we allowed age to interact with other covariates in the model. The final statistical model including this flexibility had a much lower Akaike Information Criterion compared with the model with no interactions, suggesting a better model fit. $\left(\mathrm{AIC}_{\text {Final Model }}=13,687, \mathrm{AIC}_{\text {Main effects model }}=13,785\right)$. Multicollinearity of variables in the final model was assessed using the variance inflation factor of 5. Variables in the Cox regression model included the following: age at diagnosis, gender, race, insurance coverage, year of diagnosis, stage at diagnosis, rurality, cancer group, whether the same provider performed the biopsy and the excision, whether a diagnostic test was performed by a dermatologist, whether the excision was conducted at a North Carolina National Cancer Institute Center, and the presence of 1 or more comorbid conditions. In order to assess effect heterogeneity, we looked across categories of stage at diagnosis. For this analysis, we collapsed stage at diagnosis into Stage 0, Stages IA/IB, and Stages II/ III and ran separate multivariable models to estimate survival probabilities and differences within each stratum of stage at diagnosis. We attempted to investigate cause-specific mortality; however, the frequency of events was too low to allow us to model the multivariable relationship.

\section{Results}

\section{Analytic sample}

Our analytic sample comprised 6,496 patients (Figure S1). The sample was predominantly male (58\%), non-Hispanic White (98\%), insured by Medicare (62\%), had Stage 0 disease $(50 \%)$, and had melanomas located primarily in the head/neck (31\%), upper extremities (27\%), and trunk (27\%) (Table 1). Figure 1 presents the distribution of surgical delay among the entire analytic sample; most patients had excision performed within 6 weeks of biopsy (84\%), followed by 6 weeks to 90 days (12\%), and 90-365 days after biopsy (4\%). The distribution of patient comorbidities was similar across biopsy-excision intervals (Table S1).

\section{Differences in survival probabilities}

The proportion of patients who survived after 5 years of follow-up decreased with increased time to surgery from $86.4 \%$ (less than 6 weeks) to $78.6 \%$ (greater than 90 days) (Table 1). Survival probabilities were lower with increasing surgical delay time throughout the course of follow-up (Fig. 2a). These probabilities ranged from $99.9 \%$ survival after 1 year of follow-up to $74.9 \%$ survival after 5 years of follow-up (Table 2). The absolute differences in survival probabilities $\left(S_{\text {Diff }}\right)$ for those undergoing excision $90-365$ days compared with those $<6$ weeks were moderate but increased over time: at 1 year, $\left(S_{\text {Diff: }}:-5.1 \%\right.$; $\left.95 \% \mathrm{CL}-8.0,-2.1\right)$, at 3 years $\left(S_{\text {Diff: }}:-11.6 \% ; 95 \% \mathrm{CL}-18.7,-4.6\right)$, and at 5 years of follow-up ( $S_{\text {Diff: }}-15.0 \%$; 95\% CL $\left.-24.6 \%,-5.5 \%\right)$.

The effects of excision delay varied by cancer stage (Table 3; Fig. 2a-d). Survival in stage 0 was not statistically different across biopsy to surgical intervals. Specifically, among patients with stage I disease, those whose surgical excision took place $90-365$ days after biopsy had a $-51.3 \%$ (95\% CL $-72.0 \%,-30.6 \%)$ decrease in 5 year-survival compared with those whose excision occurred $<6$ weeks. Among patients with stage II/III disease, at 1 year of followup, there was a moderate, but not statistically significant difference $(-12.8 \%$ [95\% CL $-26.0 \%, 0.4 \%])$ in survival probabilities between those with excisions 90-365 days after diagnosis vs. $<6$ weeks.

\section{Sensitivity analysis}

Our sensitivity analysis utilizing a different categorization for surgical delays greater than the 6-week guideline found similar results as our primary analysis: we observed a similar pattern where greater surgical delay interval was associated with worse survival. The most pronounced difference was among those who were treated more than 16 weeks after biopsy (data not shown).

\section{Discussion}

We assessed the association of surgical delay on overall survival. We found that patients whose surgery was performed 90-365 days after biopsy had significantly lower probabilities of survival compared with those whose surgery was performed within the guideline-recommended 6 weeks of diagnosis.

Our findings are qualitatively similar to the effect of a 90-day or longer delay on overall survival reported by Conic and colleagues [11]. In their study, the hazard ratios for allcause mortality were 1.1 (95\% CL 1.0,1.2) for 90-119 day delays and 1.1 (95\% CL 1.0, 1.2) for $\geq 120$-day surgical delays following biopsy. Similarly, among their Stage I subgroup, they reported hazard ratios of 1.3 (95\% CL 1.1, 1.5) and 1.4 (95\% CL 1.2, 1.7) for the 90-119 and $\geq 120$-day delay surgery groups. As in our current study, Conic et al. found no difference in survival for surgical delays in patients 


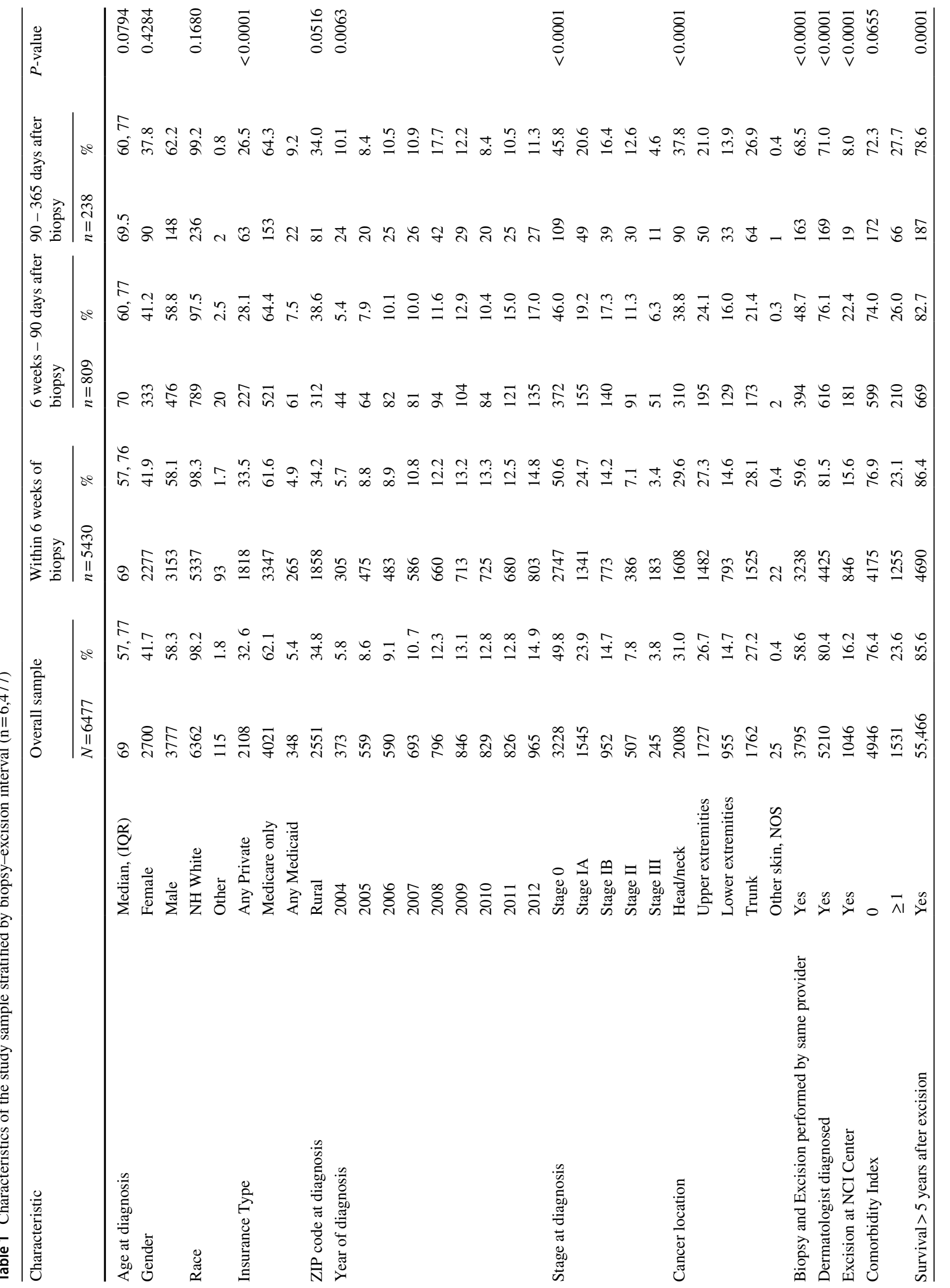


with stages II and III melanoma. Despite similar findings, their population differed in that the data used was from the National Cancer Database (NCDB) which only contains information on hospital-based patients, whereas most melanomas, particularly stage I melanomas, are diagnosed and treated in the outpatient setting [15]. Nevertheless, both our estimates of differences in survival probabilities in the overall sample as well as those diagnosed with stage I disease support evidence for the association of surgical delay on overall survival among patients with stage I melanoma, supporting evidence that timely surgery may be an important intervention to improve overall survival.

It was notable that the surgical delays were not associated with lower survival in higher stage disease. It is possible that stage I melanomas were incompletely biopsied or incompletely staged; therefore, delays in surgery resulted in further growth of residual tumor and potential for future metastasis. Estimates of monthly growth rates of invasive melanomas have been modeled between $0.11 \mathrm{~mm} / \mathrm{month}$ and $0.49 \mathrm{~mm} /$ month $[8,16]$. However, it is perhaps more plausible that our findings in early stage melanoma (Stage I) were dissimilar to findings in later stages (Stage II/III) because of small numbers in the latter group, which could have affected our results. It is also possible that patients with significant delays in surgery had disease burden not captured by the Charlson Comorbidity Index (Table 1). However, when stratifying delays by individual co-morbid conditions, disease burden is distributed similarly across biopsy-excision intervals (Table S1). While this does not prove that overall survival differences are attributed to melanoma, these findings do suggest that co-morbidity may not explain the differences entirely. Future studies analyzing the contributions of the adjusted covariates may provide further risk factor identification.

The National Academy of Medicine identified timely delivery of health care services as one of six priorities for quality health improvement in the U.S [17]. Delays affecting morbidity and mortality have also been shown in lung, bladder, and rectal cancers [18-20]. In this study, we demonstrated an association between overall survival and treatment timeliness; however, it is less clear what the factors, including patient, provider, or tumor level that could explain this association. A recent US national study of melanoma patients treated in a hospital setting showed factors associated with longer time to surgery include nonwhite race, less education, higher comorbidity burden, advanced stage, and head or neck melanoma [21]. In particular, black patients have been shown to have longer time to surgery for hospital-based melanoma [22]. Some of these delays may be explained by a higher proportion of acral lentiginous melanoma which may require coordination between unconnected specialties, worsening delays. Compared with white patients and patients with higher socioeconomic status (SES), black 
Fig. 1 Distribution of Surgical Delay
Distribution of Treatment Delay

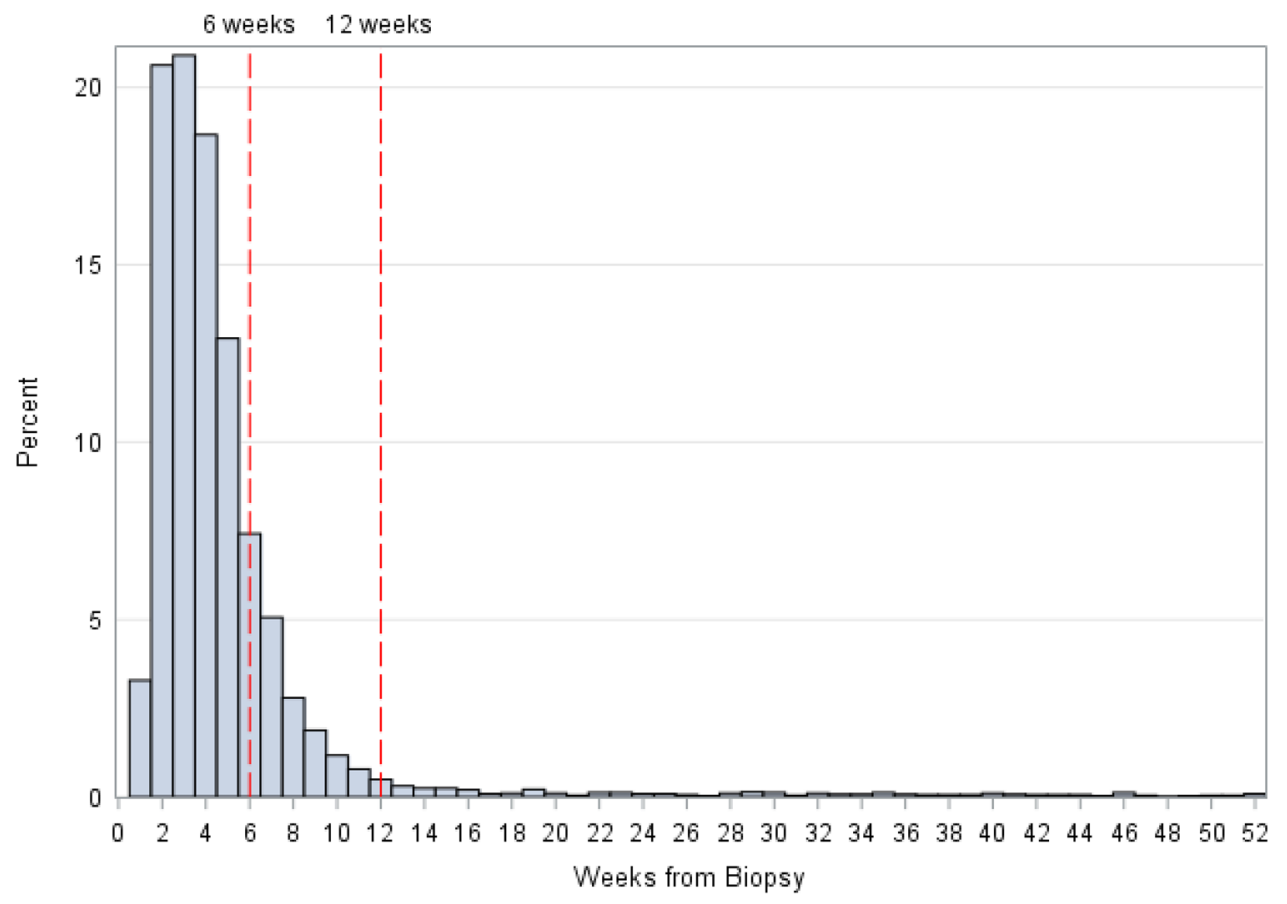

patients and poor patients have lower melanoma survival regardless of stage of disease [23, 24]. While sources of these disparities could be due to differences in tumor biology or delays in diagnosis, the lack of receipt of timely surgical care could also be a contributing factor. Therefore, further research should investigate whether potentially closing that gap in surgical delay could reduce melanoma associated disparities in outcomes, a subject of our future work.

\section{Limitations}

Our analytic sample was focused exclusively on insured patients from North Carolina diagnosed between 2004 and 2012, so our estimates of the effect may not be generalizable to other populations outside of the state. In addition, given our inclusion criteria of continuous insurance through 12 months after diagnosis, we may have missed patients who frequently cycle on and off insurance coverage. Another limitation is the relatively sparse data observed in later surgical delay categories. However, the proportion of patients with late excisions in our sample is similar to those previously reported [11]. In addition, our estimates of the effect of surgical delay on survival outcomes are based on the untestable assumptions that our models are correctly specified and there is no uncontrolled confounding. We had insufficient power to evaluate melanoma-specific survival due to the low number of observed events in our cohort; consequently, we were unable to assess whether there is an effect of surgical delay on mortality attributed to melanoma, a limitation of previous national studies [11]. Despite the inability to assess melanoma-specific survival, our findings add to the growing literature on variation in melanoma care which may adversely affect outcome [4, 11, 21, 22]. As more early stage melanomas are diagnosed over time (a significant proportion outside of the hospital setting), understanding these patterns of care will be important for potentially designing interventions to reduce disparities in melanoma outcome. Finally, because of the nature of the data and in context of our exclusion criteria, we cannot comment on surgical excisions beyond 365 days.

\section{Conclusions}

Timely surgical excision is associated with improved overall survival. Efforts should be made to ensure timeliness of surgery for patients with melanoma in order to reduce potential disparities in receipt of quality cancer care $[4,11,21,22]$. 

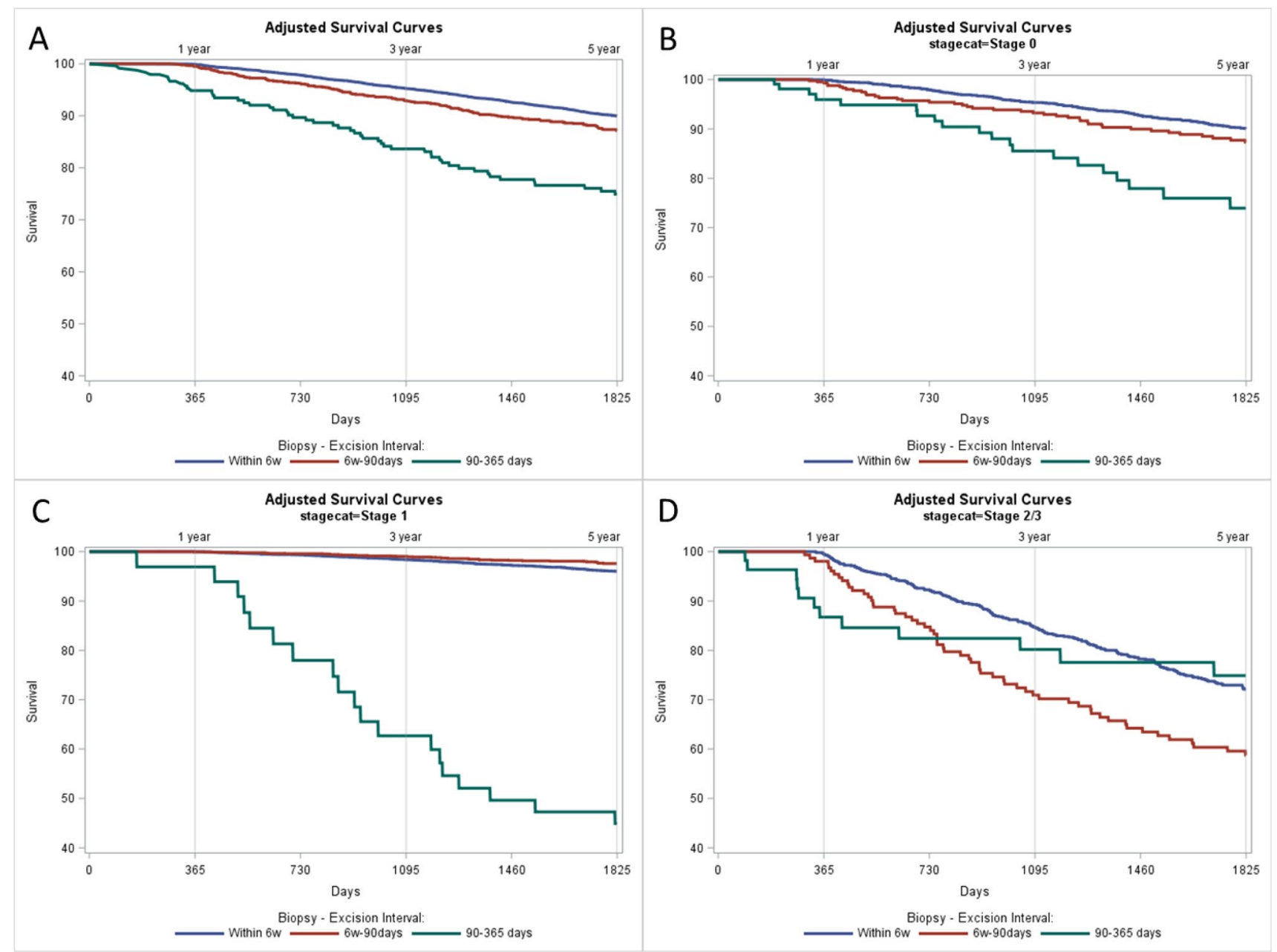

Fig. 2 Overall and stage-stratified adjusted survival probabilities according to biopsy - excision interval

Table 2 Adjusted survival probabilities and survival differences according to biopsy-excision interval $(n=6,477)$

\begin{tabular}{lllllllllllll}
\hline $\begin{array}{l}\text { Timing of } \\
\text { follow-up }\end{array}$ & $\begin{array}{l}\text { (A) } \\
<6 \text { 6wk }\end{array}$ & $\begin{array}{l}\text { (B) 6wks }- \\
90 \text { days }\end{array}$ & $\begin{array}{l}\text { (C) } \\
90-365 \text { days }\end{array}$ & $\begin{array}{l}\text { (B vs A) Sur- } \\
\text { vival difference }\end{array}$ & LCL & UCL & $\begin{array}{l}P \text {-value } \\
\text { (C vs A) } \\
\text { Survival } \\
\text { difference }\end{array}$ & LCL & UCL & $P$-value \\
\hline 1-year & 99.9 & 99.6 & 94.8 & -0.4 & -0.8 & 0.1 & 0.140 & -5.1 & -8.0 & -2.1 & $<0.001$ \\
3-year & 95.2 & 92.9 & 83.6 & -2.3 & -7.2 & 2.6 & 0.349 & -11.6 & -18.7 & -4.6 & 0.001 \\
5-year & 89.9 & 87.1 & 74.9 & -2.8 & -11.5 & 5.9 & 0.533 & -15.0 & -24.6 & -5.5 & 0.002 \\
\hline
\end{tabular}

-Biopsy-excision interval within 6 weeks is the reference group

-Survival difference $\left(S_{\text {Diff }}\right)$ adjusting for age at diagnosis, gender, race, insurance, year of diagnosis, stage at diagnosis, rurality, cancer group, whether the same provider performed the biopsy and the excision, whether a diagnostic test was performed by a dermatologist, whether excision was conducted at a North Carolina National Cancer Institute Center, and the presence of 1 or more Charlson comorbid conditions

-LCL: 95\% Lower confidence limit; UCL: 95\% upper confidence limit

-The null-value for comparison is $S_{\text {Diff }}=0$ (i.e. Survival in both groups is the same) 
Table 3 Stage-Stratified adjusted survival probabilities and survival differences according to biopsy-excision interval $(n=6,477)$

\begin{tabular}{|c|c|c|c|c|c|c|c|c|c|c|c|}
\hline $\begin{array}{l}\text { Timing of } \\
\text { follow-up }\end{array}$ & $\begin{array}{l}\text { (A) } \\
<6 \text { wks }\end{array}$ & $\begin{array}{l}\text { (B) } 6 \mathrm{wks}- \\
90 \text { days }\end{array}$ & $\begin{array}{l}\text { (C) } \\
90-365 \text { days }\end{array}$ & $\begin{array}{l}\text { (B vs A) Sur- } \\
\text { vival difference }\end{array}$ & LCL & UCL & $P$-value & $\begin{array}{l}(\mathrm{C} \text { vs } \mathrm{A}) \\
\text { Survival } \\
\text { difference }\end{array}$ & LCL & UCL & $P$-value \\
\hline \multicolumn{12}{|l|}{ Stage 0} \\
\hline 1-year & 99.9 & 99.5 & 95.9 & -0.4 & -1.4 & 0.6 & 0.393 & -4.0 & -8.6 & 0.6 & 0.090 \\
\hline 3-year & 95.3 & 93.2 & 85.5 & -2.1 & -13.4 & 9.2 & 0.715 & -9.8 & -23.5 & 3.9 & 0.160 \\
\hline 5-year & 90.0 & 87.3 & 74.0 & -2.7 & -23.4 & 18.1 & 0.802 & -16.0 & -34.3 & 2.2 & 0.085 \\
\hline \multicolumn{12}{|l|}{ Stage I } \\
\hline 1-year & 99.9 & 99.9 & 96.9 & -0.0 & -0.2 & 0.1 & 0.651 & -3.1 & -8.8 & 2.7 & 0.292 \\
\hline 3 -year & 98.4 & 99.0 & 62.5 & 0.7 & -1.9 & 3.3 & 0.616 & -35.7 & -54.2 & -17.3 & $<0.001$ \\
\hline 5-year & 96.0 & 97.6 & 44.7 & 1.6 & -4.7 & 8.0 & 0.616 & -51.3 & -72.0 & -0.6 & $<0.001$ \\
\hline \multicolumn{12}{|l|}{ Stage II/III } \\
\hline 1-year & 99.5 & 98.0 & 86.7 & -1.5 & -4.3 & 1.4 & 0.314 & -12.8 & -26.0 & 0.4 & 0.057 \\
\hline 3-year & 84.7 & 70.9 & 80.2 & -13.8 & -35.2 & 7.5 & 0.210 & -4.6 & -22.3 & 13.4 & 0.616 \\
\hline 5-year & 72.3 & 58.8 & 74.9 & -13.5 & -41.2 & 14.5 & 0.348 & 2.6 & -19.9 & 25.2 & 0.819 \\
\hline
\end{tabular}

-Biopsy-excision interval within 6 weeks is the reference group

-Survival difference $\left(S_{\text {Diff }}\right)$ adjusting for age at diagnosis, gender, race, insurance, year of diagnosis, rurality, cancer group, whether the same provider performed the biopsy and the excision, whether a diagnostic test by dermatologist, whether excision was conducted at a North Carolina National Cancer Institute Center, and the presence of 1 or more Charlson comorbid conditions

-LCL: 95\% Lower confidence limit; UCL: 95\% upper confidence limit

-The null-value for comparison is $S_{\text {Diff }}=0$ (i.e. Survival in both groups is the same)

Funding Dr. Adamson is supported by the Dermatology Foundation Public Health Career Development Award and by the National Institutes of Health under grant NIH UL1 TR002645. The funding sources had no role in the design and conduct of the study; collection, management, analysis, and interpretation of the data; preparation, review, or approval of the manuscript; and decision to submit the manuscript for publication.

\section{Compliance with ethical standards}

Conflict of interest None reported.

IRB statement The University of North Carolina at Chapel Hill IRB approved this study.

\section{References}

1. SEER*Stat Database: (1975-2016). Bethesda, MD: National Cancer Institute Surveillance Research Program, 2019 (https://www. seer.cancer.gov)

2. Ekwueme DU, Guy GP Jr, Li C, Rim SH, Parelkar P, Chen SC (2011) The health burden and economic costs of cutaneous melanoma mortality by race/ethnicity-United States, 2000 to 2006. J Am Acad Dermatol 65(5 Suppl 1):S133-143

3. Bleicher RJ, Ruth K, Sigurdson ER et al (2016) Time to Surgery and Breast Cancer Survival in the United States. JAMA Oncol 2(3):330-339

4. Adamson AS, Zhou L, Baggett CD, Thomas NE, Meyer AM (2017) Association of delays in surgery for melanoma with insurance type. JAMA Dermatol 153(11):1106-1113. https://doi. org/10.1001/jamadermatol.2017.3338

5. Lott JP, Narayan D, Soulos PR, Aminawung J, Gross CP (2015) Delay of surgery for melanoma among medicare beneficiaries. JAMA Dermatol 151(7):731-741

6. Huff LS, Chang CA, Thomas JF et al (2012) Defining an acceptable period of time from melanoma biopsy to excision. Dermatol Rep 4(1):e2

7. Garbe C, Peris K, Hauschild A et al (2016) Diagnosis and treatment of melanoma. European consensus-based interdisciplinary guideline - Update 2016. Eur J Cancer. 63:201-217

8. Lin MJ, Mar V, McLean C, Kelly JW (2014) An objective measure of growth rate using partial biopsy specimens of melanomas that were initially misdiagnosed. J Am Acad Dermatol 71(4):691-697

9. McKenna DB, Lee RJ, Prescott RJ, Doherty VR (2002) The time from diagnostic excision biopsy to wide local excision for primary cutaneous malignant melanoma may not affect patient survival. Br J Dermatol 147(1):48-54

10. Carpenter S, Pockaj B, Dueck A et al (2008) Factors influencing time between biopsy and definitive surgery for malignant melanoma: do they impact clinical outcome? Am J Surg. 196(6):834-842

11. Conic RZ, Cabrera CI, Khorana AA, Gastman BR (2018) Determination of the impact of melanoma surgical timing on survival using the National Cancer Database. J Am Acad Dermatol. 78(1):40-46

12. Meyer AM, Olshan AF, Green L et al (2014) Big data for population-based cancer research: the integrated cancer information and surveillance system. N C Med J 75(4):265-269

13. Hart LG, Larson EH, Lishner DM (2005) Rural definitions for health policy and research. Am J Public Health 95(7):1149-1155

14. Howe CJ, Cole SR, Westreich DJ, Greenland S, Napravnik S, Eron JJ Jr (2011) Splines for trend analysis and continuous confounder control. Epidemiology 22(6):874-875 
15. Bilimoria KY, Stewart AK, Winchester DP, Ko CY (2008) The National Cancer Data Base: a powerful initiative to improve cancer care in the United States. Ann Surg Oncol 15(3):683-690

16. Liu W, Dowling JP, Murray WK et al (2006) Rate of growth in melanomas: characteristics and associations of rapidly growing melanomas. Arch Dermatol 142(12):1551-1558

17. Institute of Medicine (U.S.).(2001) Committee on Quality of Health Care in America. Crossing the quality chasm: a new health system for the 21 st century. Washington, D.C.: National Academy Press

18. Bilimoria KY, Ko CY, Tomlinson JS et al (2011) Wait times for cancer surgery in the United States: trends and predictors of delays. Ann Surg 253(4):779-785

19. Korsgaard M, Pedersen L, Sorensen HT, Laurberg S (2006) Delay of treatment is associated with advanced stage of rectal cancer but not of colon cancer. Cancer Detect Prev 30(4):341-346

20. Bardell T, Belliveau P, Kong W, Mackillop WJ (2006) Waiting times for cancer surgery in Ontario: 1984-2000. Clin Oncol (R Coll Radiol) 18(5):401-409

21. Baranowski MLH, Yeung H, Chen SC, Gillespie TW, Goodman M (2019) Factors associated with time to surgery in melanoma: An analysis of the National Cancer Database. J Am Acad Dermatol 81(4):908-916

22. Tripathi R, Archibald LK, Mazmudar RS, Conic RRZ, Rothermel LD, Scott JF, Bordeaux JS (2020) Racial differences in time to treatment for melanoma. J Am Acad Dermatol 83(3):854-859. https://doi.org/10.1016/j.jaad.2020.03.094

23. Dawes SM, Tsai S, Gittleman H, Barnholtz-Sloan JS, Bordeaux JS (2016) Racial disparities in melanoma survival. J Am Acad Dermatol 75(5):983-991

24. Sitenga JL, Aird G, Ahmed A, Walters R, Silberstein PT (2018) Socioeconomic status and survival for patients with melanoma in the United States: an NCDB analysis. Int J Dermatol 57(10):1149-1156

Publisher's Note Springer Nature remains neutral with regard to jurisdictional claims in published maps and institutional affiliations. 